\title{
High Resolution Estimation of AoA, AoD and TdoA from MIMO Channel Sounding Measurements with Virtual Antenna Arrays: Maximum-Likelihood vs. Unitary Tensor-ESPRIT
}

\author{
Stephan Häfner and Reiner Thomä
}

\begin{abstract}
Estimating the parameters of a geometric propagation model from MIMO channel sounding measurements will be considered, which requires the solution of an inverse problem. Thus, a model of the measured data is derived, which incorporates a model of the measurement system as well as the parameters of interest. Based on the data model a maximum-likelihood estimator will be derived to infer the model parameters. Because virtual antenna arrays are considerer, formed by step-wise rotating directive antennas at transmitter and receiver side, the MIMO measurements are conducted in the beam-space. Hence, the data model can be described by a multidimensional convolution of the measurement system and the propagation channel. Based on the convolutional modelling, the parameter estimation problem is transformed into a harmonic retrieval problem, which can be solved by an Unitary Tensor-ESPRIT algorithm. The maximum-likelihood and ESPRIT estimator are compared by Monte-Carlo simulations according to their rootmean-square estimation error.
\end{abstract}

Keywords-MIMO Measurements, Propagation Channel Model, Inverse Problem, Parameter Estimation, MaximumLikelihood, Beam-space Processing, Rotational Invariance, Harmonic Retrieval

\section{Introduction}

C HANNEL sounding, the empirical investigation of wave propagation by measuring a channel impulse response (CIR), is a prerequisite to understand the characteristics of the propagation channel [1]. Knowledge of channel characteristics is required to e.g. develop new air interfaces or to derive channel models. Because novel air interfaces are considered to be equipped with multiple antennas, the propagation channel has to be characterised in temporal and spatial domain, hence multidimensional sounding is conducted.

De-embedding of the channel sounder system from the measurements is required to gain access on the raw propagation channel. The de-embedding of the measurement system is possible by model-based identification of the system of interest (the propagation channel) from the measurements, while knowing the measurement system in terms of a system model. Therefore, parametric system identification is employed and geometrical parameters like azimuth of arrival (AoA), azimuth of departure (AoD) or time difference of arrival (TdoA) of

Manuscript received December 12, 2017; revised June 21, 2018.

Stephan Häfner and Reiner Thomä are with the Institute for Information Technology, Technische Universität Ilmenau, 98684 Ilmenau, Germany, email:(stephan.haefner,reiner.thomae)@tu-ilmenau.de. a geometrical propagation channel model [2] are estimated from the measurements. Parameter estimation or parameter inference is an inverse problem, which requires a data model to describe the mapping of the parameters of interest to the measured data. Afterwards, parameter estimators are applied to solve the inverse problem.

Parametric or model-based system identification provides a much higher resolution than non-parametric methods. Here, resolution is denoted as the capability to separate e.g. two propagation paths in a certain measurement dimension. Resolution of non-parametric methods is mainly determined by the aperture size of the measurement system in the respective dimension, known as the Rayleigh resolution limit. Modelbased estimation can overcome this resolution limit, why such methods are denoted as high resolution parameter estimation (HRPE). Resolution of such methods is mainly limited by the available signal to noise ratio (SNR) and the model accuracy, but also the finite device aperture as e.g. bandwidth, array size and observation time.

For spatial channel characterisation and in order to estimate the model parameters AoA and AoD, multiple input multiple output (MIMO) measurements with antenna arrays at both link ends are commonly conducted [1]. Utilising antenna arrays at both sides is the most promising approach, because it allows a fast multidimensional acquisition of the propagation channel [1], which is required for e.g. time-variant propagation channels. By step-wise rotating a single antenna at transmitter (Tx) and receiver ( $\mathrm{Rx})$ side, which is quite often employed for channel sounding at millimetre wave frequencies [3], [4], virtual (synthetic) antenna arrays are formed at both link ends. Such mechanical arrangements are more ease to construct than real antenna arrays, to the cost of a much longer measurement duration. Consequently, the stationariness of the propagation channel has to be much higher compared to array based measurements, why this approach seems to be suitable for timeinvariant propagation channels only. The rotated antennas can have omni-directional characteristics, which coincides with a pure spatial channel sampling; or the antenna can be directive, which coincides with a measurement in the beam-space [5]. In this paper, beam-space measurements with directive antennas are considered.

Algorithms to estimate the propagation model parameters from multidimensional sounding measurement with real arrays [6] and virtual antenna array [7]-[11] are known from lit- 
erature. Quite often, estimators of maximum-likelihood (ML) type are utilised, where a cost function based on the measured data's distribution is defined. This cost function has to be optimised w.r.t. the model parameters, which can become computationally cumbersome due to the high dimensionality. Also, convergence to a global optimum is hard to guarantee because the cost functions are typically non-convex.

The step-wise rotation of antennas allows to formulate the data model as a multi-dimensional convolution of the propagation channel and the channel sounding system. By applying beam-space transformation and subsequent multidimensional deconvolution with the measurement system response, the parameter estimation problem is transformed into a 3-dimensional harmonic retrieval problem. Considerable solvers for such a problem are e.g. the multi-dimensional MUSIC [12], the multi-dimensional rank reduction estimator (RARE) [13] as well as multi-dimensional PARAFAC [14] Here, the Unitary Tensor-ESPRIT [15], [16] will be utilised. The Estimation of Signal Parameters via Rotational Invariance Technique (ESPRIT) algorithm avoids costly enumerativebased search and global convergence is guaranteed, to the cost of possibly degraded estimation accuracy due to a simplified data model. Estimation performance of the ESPRIT and the ML will be compared by Monte-Carlo simulations.

The paper is structured as follows: the data model for the inverse problem is introduced in Section II. The ML estimator is derived in Section III. Beam-space transformation and application of the Unitary Tensor-ESPRIT is explained in Section IV. Simulation based comparison of ML and ESPRIT is presented in Section V. Section VI concludes the paper.

\section{A. Mathematical Notation}

The following notation is used to indicate scalars, vectors, matrices and tensors: Scalars are italic letters $(m, \ldots, M, \ldots, \tau)$, column vectors are written as bold-face lower-case letters $(\mathbf{a}, \ldots, \boldsymbol{\tau})$, matrices correspond to bold face upper-case letters $(\mathbf{A}, \ldots, \boldsymbol{\Sigma})$, and tensors are written as bold-face calligraphic letters $(\mathcal{Y}, \mathcal{X}, \ldots)$.

Convolution of two functions according to their matching dimensions is denoted by the $*$ operator. The matrix operations $(\bullet)^{*},(\bullet)^{T},(\bullet)^{\dagger}$ and $(\bullet)^{H}$ are defined as the conjugate, transpose, Moore-Penrose pseudo-inverse and conjugate transpose, respectively. Moreover, the Kronecker product of two matrices is symbolised by $\otimes$ and the Khatri-Rao product (column-wise Kronecker product) by $\diamond$.

\section{Measurement Data Model and Inverse Problem}

In order to infer parameter values, a model of the measurement data is required. This model incorporates the measurement system and the propagation channel. Basically, the measurement system is described by a model of the antenna array. The propagation channel is described in terms of the propagation parameters AoA, AoD and TdoA.

\section{A. Propagation Channel Model}

The propagation channel is assumed as the superposition of multiple propagating waves or paths. Each path is modelled

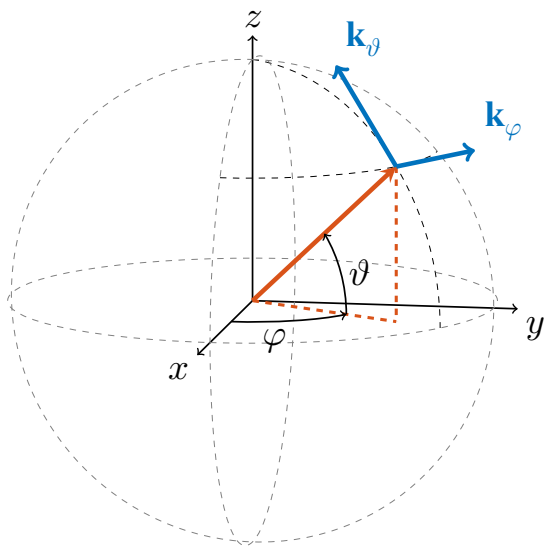

Fig. 1: Spherical coordinate system and polarisation definition

as a discrete optical ray, describing a delayed transmission from $\mathrm{Tx}$ to $\mathrm{Rx}$ in the delay domain [2]. Propagation in the spatial domain is described in terms of the angles AoA and elevation of arrival (EoA) at the Rx, and AoD and elevation of departure (EoD) at the Tx. The elevation angle $\vartheta$ is defined in the range from $[-\pi / 2, \pi / 2]$ and the azimuth angle $\varphi$ in the range of $[-\pi, \pi]$, see Fig. 1. Polarisation of a wave is defined according to the $\varphi$ - $\vartheta$-plane, spanned by the spherical coordinate system basis vectors $\mathbf{k}_{\varphi}$ and $\mathbf{k}_{\vartheta}$ in the impingement point on the sphere. Because of the assumed discrete nature of each path, the model is a superposition of Dirac deltas in the angular-delay domain. Hence, the model of the time invariant propagation channel is given by [17]

$$
\begin{aligned}
\mathbf{h}\left(\boldsymbol{\kappa}^{T x}, \boldsymbol{\kappa}^{R x}, \tau\right) \\
\quad=\sum_{p=1}^{P} \boldsymbol{\gamma}_{p} \cdot \delta\left(\boldsymbol{\kappa}^{T x}-\boldsymbol{\kappa}_{p}^{T x}\right) \cdot \delta\left(\boldsymbol{\kappa}^{R x}-\boldsymbol{\kappa}_{p}^{R x}\right) \cdot \delta\left(\tau-\tau_{p}\right)
\end{aligned}
$$

with $\boldsymbol{\kappa}_{p}^{R x}=\left[\begin{array}{ll}\varphi_{p}^{R x} & \vartheta_{p}^{R x}\end{array}\right]^{T}$ and $\boldsymbol{\kappa}_{p}^{T x}=\left[\begin{array}{ll}\varphi_{p}^{T x} & \vartheta_{p}^{T x}\end{array}\right]^{T}$ containing the azimuth and elevation angles at $\mathrm{Rx}$ and $\mathrm{Tx}$ side, respectively. The propagation delay or TdoA is denoted by $\tau_{p}$ and $\gamma_{p}=\left[\begin{array}{llll}\gamma_{p}^{\varphi, \varphi} & \gamma_{p}^{\varphi, \vartheta} & \gamma_{p}^{\vartheta, \varphi} & \gamma_{p}^{\vartheta, \vartheta}\end{array}\right]^{T}$ is the vector of polarimetric path weights.

\section{B. Measurement System Model}

1) Virtual Array Model: An antenna is assumed as linear, time invariant system with a finite impulse response duration. Hence, the antenna's angle and polarisation dependent impulse response $\mathbf{b}_{0}(\varphi, \vartheta, \tau)$ can be utilised to describe the antenna's radiation properties in the far-field, denoted by vector $\mathbf{e}(\varphi, \vartheta, \tau)$.

$$
\mathbf{e}(\varphi, \vartheta, \tau)=\left[\mathbf{k}_{\varphi} \mathbf{k}_{\vartheta}\right]\left[\begin{array}{l}
b_{0}^{\varphi}(\varphi, \vartheta, \tau) \\
b_{0}^{\vartheta}(\varphi, \vartheta, \tau)
\end{array}\right]=\mathbf{K} \cdot \mathbf{b}_{0}(\varphi, \vartheta, \tau)
$$

The propagation channel is sampled in the spatial domain by an array of antennas. Here, a virtual uniform circular array (UCA) is considered, formed by step-wise rotating a single antenna w.r.t the origin of a spherical coordinate system. Because the azimuth plane is sampled only, the angular domain is limited to the azimuth domain and subsequent investigations 
are limited to AoA and AoD. Elevation angles are assumed as $0^{\circ}$. Rotating a single antenna is an angular invariant process, as long as the antenna pattern does not change during the rotation [18]. Assuming angular invariance, the polarimetric array response $\mathbf{b}(\varphi, \tau)$ is given by the convolution of the antenna's polarimetric impulse response and the direction of impingement $\varphi_{p}$.

$$
\mathbf{b}(\varphi, \tau)=\mathbf{b}_{0}\left(\varphi-\varphi_{p}, \tau\right)=\mathbf{b}_{0}(\varphi, \tau) * \delta\left(\varphi-\varphi_{p}\right)
$$

By rotating the antenna to a set of $M$ discrete angles in the range from $\left[0,2 \pi-\varphi_{0}\right]$, the convolution result is sampled at $m=0 \ldots M-1$ antenna positions [5]. Parameter $\varphi_{0}$ denotes the rotation step size.

$$
\mathbf{b}\left(m \varphi_{0}, \tau\right)=\mathbf{b}_{0}\left(m \varphi_{0}-\varphi_{p}, \tau\right)
$$

The sampling results are concatenated in matrix $\mathbf{B}\left(\varphi_{p}, \tau\right) \in \mathbb{C}^{2 \times M}$, denoted as the array response matrix.

$$
\begin{aligned}
& \mathbf{B}\left(\varphi_{p}, \tau\right)=\left[\begin{array}{lll}
\mathbf{b}_{0}\left(-\varphi_{p}, \tau\right) & \ldots \mathbf{b}_{0}\left((M-1) \varphi_{0}-\varphi_{p}, \tau\right)
\end{array}\right] \\
& =\left[\begin{array}{lll}
b_{0}^{\varphi}\left(-\varphi_{p}, \tau\right) & \ldots & b_{0}^{\varphi}\left((M-1) \varphi_{0}-\varphi_{p}, \tau\right) \\
b_{0}^{\vartheta}\left(-\varphi_{p}, \tau\right) & \ldots & b_{0}^{\vartheta}\left((M-1) \varphi_{0}-\varphi_{p}, \tau\right)
\end{array}\right]
\end{aligned}
$$

Considering dual-polarimetric antennas, hence the rotated antenna features two ports which are orthogonally polarised, the array response matrix has to be extended accordingly. The array response matrix $\mathbf{B}_{\text {polar }}\left(\varphi_{p}, \tau\right) \in \mathbb{C}^{2 \times 2 M}$ for dualpolarimetric antennas is introduced, built by

$$
\begin{aligned}
\mathbf{B}_{\text {polar }}\left(\varphi_{p}, \tau\right) & =\left[\begin{array}{lll}
\mathbf{B}_{1}\left(\varphi_{p}, \tau\right) & \ldots & \mathbf{B}_{2}\left(\varphi_{p}, \tau\right)
\end{array}\right] \\
& =\left[\begin{array}{lll}
\mathbf{b}_{\text {polar }}^{\varphi}\left(\varphi_{p}, \tau\right) & \mathbf{b}_{\text {polar }}^{\vartheta}\left(\varphi_{p}, \tau\right)
\end{array}\right]^{T}
\end{aligned}
$$

Matrix $\mathbf{B}_{1}\left(\varphi_{p}, \tau\right)$ and $\mathbf{B}_{2}\left(\varphi_{p}, \tau\right)$ denote the array response matrix for the respective antenna ports, built according to equation (5). Vectors $\mathbf{b}_{\text {polar }}^{\varphi}\left(\varphi_{p}, \tau\right) \in \mathbb{C}^{2 M \times 1}$ and $\mathbf{b}_{\text {polar }}^{\vartheta}\left(\varphi_{p}, \tau\right) \in \mathbb{C}^{2 M \times 1}$ denote the array response according to the respective polarisation.

2) Noise Model: Another part of the measurement system model is the noise model. Here, the noise is assumed as an additive, complex circular, random process; with zero-mean and Gaussian distribution [19], denoted by $\mathbf{n} \sim \mathcal{C N}(\mathbf{0} ; \boldsymbol{\Sigma})$. The noise model is parametrised by the covariance matrix $\boldsymbol{\Sigma}$, which depends on the noise covariance $\boldsymbol{\Sigma}_{T x}$ at Tx, $\boldsymbol{\Sigma}_{R x}$ at $\mathrm{Rx}$ and $\boldsymbol{\Sigma}_{\tau}$ in the delay domain. Assuming the noise processes in these dimensions being uncorrelated, the noise covariance matrix comprises a Kronecker structure.

$$
\boldsymbol{\Sigma}=\boldsymbol{\Sigma}_{\tau} \otimes \boldsymbol{\Sigma}_{T x} \otimes \boldsymbol{\Sigma}_{R x}
$$

Additionally, the noise is assumed as spatially and temporarily uncorrelated. Hence, the noise covariance matrix becomes

$$
\boldsymbol{\Sigma}(\sigma)=\sigma^{2} \cdot \mathbf{I}_{M_{f}} \otimes \mathbf{I}_{M_{t}} \otimes \mathbf{I}_{M_{r}}
$$

with $\sigma^{2}$ denoting the noise power.

\section{Measurement Data Model}

Considering the introduced propagation and measurement system model, the data model is the multi-dimensional convolution of the propagation channel model and the antenna response at Tx and Rx side, plus additive Gaussian noise $n(\tau)$.

$$
\begin{aligned}
& y\left(\varphi^{R x}, \varphi^{T x}, \tau\right)=m\left(\varphi^{R x}, \varphi^{T x}, \tau\right)+n(\tau) \\
& \quad=\mathbf{h}^{T}\left(\varphi^{R x}, \varphi^{T x}, \tau\right) * \mathbf{b}_{0}\left(\varphi^{R x}, \varphi^{T x}, \tau\right)+n(\tau)
\end{aligned}
$$

Note, that the propagation channel model is reduced to the case of $0^{\circ}$ EoA and EoD. Vector $\mathbf{b}_{0}\left(\varphi^{R x}, \varphi^{T x}, \tau\right)$ denotes the measurement system response, which is given by the convolution of the polarimetric antenna response of Tx and Rx antenna.

$$
\begin{aligned}
& \mathbf{b}_{0}\left(\varphi^{R x}, \varphi^{T x}, \tau\right) \\
& \quad=\int \mathbf{b}_{0, R x}\left(\varphi^{R x}, \epsilon\right) \otimes \mathbf{b}_{0, T x}\left(\varphi^{T x}, \tau-\epsilon\right) d \epsilon
\end{aligned}
$$

The multi-dimensional convolution in equation (9) is sampled in azimuth by rotating the antenna at $\mathrm{Tx}$ and $\mathrm{Rx}$ to $M_{t}$ and $M_{r}$ positions with step size $\varphi_{0}^{T x}$ and $\varphi_{0}^{R x}$, respectively. The delay domain is sampled at $M_{f}$ points with step size $\tau_{0}$.

Assuming dual-polarimetric antennas at both link ends, 4 polarisation combinations are given for each combination of Tx and Rx antenna positions. The corresponding measurements are concatenated in the tensor $\mathcal{Y} \in \mathbb{C}^{2 M_{r} \times 2 M_{t} \times M_{f}}$.

\section{The Inverse Problem}

The parameters AoA, AoD, TdoA and polarimetric paths weights for all paths are concatenated in the vectors $\varphi^{R x}$, $\varphi^{T x}, \tau$ and $\gamma$.

$$
\begin{array}{rlrl}
\varphi^{R x} & =\left[\begin{array}{lll}
\varphi_{1}^{R x} & \ldots & \varphi_{P}^{R x}
\end{array}\right]^{T} & & \in \mathbb{C}^{P \times 1} \\
\varphi^{T x} & =\left[\begin{array}{lll}
\varphi_{1}^{T x} & \ldots & \varphi_{P}^{T x}
\end{array}\right]^{T} & & \in \mathbb{C}^{P \times 1} \\
\boldsymbol{\tau} & =\left[\begin{array}{lll}
\tau_{1} & \ldots & \tau_{P}
\end{array}\right]^{T} & & \in \mathbb{C}^{P \times 1} \\
\gamma & =\left[\begin{array}{llll}
\gamma^{\varphi, \varphi^{T}} & \gamma^{\varphi, \vartheta^{T}} & \gamma^{\vartheta, \varphi^{T}} & \gamma^{\vartheta, \vartheta^{T}}
\end{array}\right]^{T} & \in \mathbb{C}^{4 P \times 1}
\end{array}
$$

The parameter estimation task is to deduce the vectors $\varphi^{R x}$, $\varphi^{T x}, \tau, \gamma$ from the measurements $\mathcal{Y}$. Based on the data model (9), mapping of the parameters to the measurement data is known.

$$
m: \varphi^{R x}, \varphi^{T x}, \boldsymbol{\tau}, \gamma, \sigma \rightarrow \mathcal{Y}
$$

Because the parameters have to be inferred from the measurements, an inverse problem is given.

$$
m^{-1}: \mathcal{Y} \rightarrow \varphi^{R x}, \varphi^{T x}, \boldsymbol{\tau}, \gamma, \sigma
$$

Note, that the noise model parameter $\sigma$ is not of immediate interest, but have to be taken into account during parameter estimation. This kind of parameters are denoted as nuisance parameters. 


\section{Maximum-Likelihood Estimator}

\section{A. Sampled Data Model}

For concise description, the data model in equation (9) is re-written in matrix-vector notation. First, the data model is transformed to frequency domain $(\tau \rightarrow f)$, which yields

$$
\mathbf{y}=\operatorname{vec}\left\{[\mathcal{Y}]_{(1)}\right\}=\mathbf{M}\left(\varphi^{R x}, \varphi^{T x}, \boldsymbol{\tau}\right) \cdot \boldsymbol{\gamma}+\mathbf{n} \quad \in \mathbb{C}^{4 M \times 1}
$$

with $[\bullet]_{(1)}$ the 1-mode unfolding [20] of the measurement tensor and vec $\{\bullet\}$ the vectorising operator, stacking the matrix columns. Matrix $\mathbf{M}\left(\varphi^{R x}, \varphi^{T x}, \boldsymbol{\tau}\right) \in \mathbb{C}^{4 M \times 4 P}$ contains the sampled model function $m\left(\varphi^{R x}, \varphi^{T x}, \tau\right)$ in equation (9) for $P$ paths and all polarisation combinations.

$$
\begin{aligned}
\mathbf{M}\left(\varphi^{R x}, \varphi^{T x}, \boldsymbol{\tau}\right) & \\
= & \mathbf{M}^{\varphi, \varphi}\left(\varphi^{R x}, \varphi^{T x}, \boldsymbol{\tau}\right) \mathbf{M}^{\varphi, \vartheta}\left(\varphi^{R x}, \varphi^{T x}, \boldsymbol{\tau}\right) \ldots \\
& \left.\mathbf{M}^{\vartheta, \varphi}\left(\varphi^{R x}, \varphi^{T x}, \boldsymbol{\tau}\right) \mathbf{M}^{\vartheta, \vartheta}\left(\varphi^{R x}, \varphi^{T x}, \boldsymbol{\tau}\right)\right]
\end{aligned}
$$

Accordingly, matrix $\mathbf{M}^{\alpha, \beta}\left(\varphi^{R x}, \varphi^{T x}, \boldsymbol{\tau}\right) \in \mathbb{C}^{4 M \times P}$ contains the sampled model function $m\left(\varphi^{R x}, \varphi^{T x}, \tau\right)$ for $P$ paths and polarisation combination $\alpha, \beta$. The variables $\alpha, \beta$ denote either $\varphi$ or $\vartheta$ polarisation.

$$
\begin{aligned}
& \mathbf{M}^{\alpha, \beta}\left(\boldsymbol{\varphi}^{R x}, \varphi^{T x}, \boldsymbol{\tau}\right) \\
& \quad=\left[\mathbf{m}^{\alpha, \beta}\left(\varphi_{1}^{R x}, \varphi_{1}^{T x}, \tau_{1}\right) \ldots \mathbf{m}^{\alpha, \beta}\left(\varphi_{P}^{R x}, \varphi_{P}^{T x}, \tau_{P}\right)\right]
\end{aligned}
$$

The vector $\mathbf{m}^{\alpha, \beta}\left(\varphi_{p}^{R x}, \varphi_{p}^{T x}, \tau_{p}\right) \in \mathbb{C}^{4 M \times 1}$ represents the sampled model function $m\left(\varphi^{R x}, \varphi^{T x}, \tau\right)$ for one path and polarisation combination $\alpha, \beta$.

$$
\begin{aligned}
\mathbf{m}^{\alpha, \beta}\left(\varphi_{p}^{R x}, \varphi_{p}^{T x}, \tau_{p}\right) \\
\quad=\operatorname{vec}\left\{\mathbf{a}^{T}\left(\tau_{p}\right) \diamond \mathbf{B}_{T x}^{\alpha}\left(\varphi_{p}^{T x}\right) \diamond \mathbf{B}_{R x}^{\beta}\left(\varphi_{p}^{R x}\right)\right\} .
\end{aligned}
$$

The sampling vector contains the matrices $\mathbf{B}_{T x}^{\alpha}\left(\varphi_{p}^{T x}\right) \in \mathbb{C}^{2 M_{t} \times M_{f}}$ and $\mathbf{B}_{R x}^{\beta}\left(\varphi_{p}^{R x}\right) \in \mathbb{C}^{2 M_{r} \times M_{f}}$, comprising the frequency response of the virtual UCA at Tx and $\mathrm{Rx}$, sampled at $M_{f}$ frequency bins.

$$
\begin{aligned}
& \mathbf{B}_{R x}^{\alpha}\left(\varphi_{p}^{R x}\right)=\left[\begin{array}{llll}
\mathbf{b}_{R x}^{\alpha}\left(\varphi_{p}^{R x}, 0\right) & \ldots & \mathbf{b}_{R x}^{\alpha}\left(\varphi_{p}^{R x},\left(M_{f}-1\right) f_{0}\right)
\end{array}\right] \\
& \mathbf{B}_{T x}^{\beta}\left(\varphi_{p}^{T x}\right)=\left[\begin{array}{llll}
\mathbf{b}_{T x}^{\beta}\left(\varphi_{p}^{T x}, 0\right) & \ldots & \mathbf{b}_{T x}^{\beta}\left(\varphi_{p}^{T x},\left(M_{f}-1\right) f_{0}\right)
\end{array}\right]
\end{aligned}
$$

Vector $\mathbf{b}_{R x}^{\alpha}\left(\varphi_{p}^{R x}, m_{f} f_{0}\right)$ and $\mathbf{b}_{T x}^{\beta}\left(\varphi_{p}^{T x}, m_{f} f_{0}\right)$ are the array response vectors in frequency domain, according to equation (6). Vector $\mathbf{a}\left(\tau_{p}\right) \in \mathbb{C}^{M_{f} \times 1}$ contains the exponentials according to the delay.

$$
\mathbf{a}\left(\tau_{p}\right)=\left[1 \ldots \exp \left(-\jmath 2 \pi\left(M_{f}-1\right) f_{0} \tau_{p}\right)\right]^{T}
$$

\section{B. Cost Function Derivation}

Based on the noise model assumption, the distribution of the measurement vector $\mathbf{y}$ is

$$
\mathbf{y} \sim \mathcal{C N}\left(\mathbf{M}\left(\varphi^{R x}, \varphi^{T x}, \boldsymbol{\tau}\right) \cdot \boldsymbol{\gamma} ; \boldsymbol{\Sigma}(\sigma)\right)
$$

Using the ML method [21], the likelihood function $f(\mathbf{y} \mid \boldsymbol{\theta})$ with $\boldsymbol{\theta}=\left[\boldsymbol{\varphi}^{R x^{T}} \boldsymbol{\varphi}^{T x^{T}} \boldsymbol{\tau}^{T} \gamma^{T} \sigma\right]^{T}$ has to be maximised to deduce parameter values. For sake of convenience, the arguments are dropped.

$$
f(\mathbf{y} \mid \boldsymbol{\theta})=\frac{\exp \left(-(\mathbf{y}-\mathbf{M} \cdot \boldsymbol{\gamma})^{H} \boldsymbol{\Sigma}^{-1}(\mathbf{y}-\mathbf{M} \cdot \boldsymbol{\gamma})\right)}{\operatorname{det}\{\pi \boldsymbol{\Sigma}\}}
$$

Plugging in the noise model (8), taking the natural logarithm of the likelihood function and neglecting constant terms yields the cost function for parameter estimation

$$
\mathcal{L}=2 M \ln (\sigma)+\sigma^{-2}(\mathbf{y}-\mathbf{M} \cdot \boldsymbol{\gamma})^{H}(\mathbf{y}-\mathbf{M} \cdot \boldsymbol{\gamma})
$$

This cost function has to be minimised for parameter estimation purpose. The framework presented in [22] is used therefore.

\section{Beam-Space Unitary Tensor-ESPRIT}

The discrete Fourier transform is used for beam-space transformation of the measurements in azimuth and delay domain. Based on the beam-space transformed measurements, the Unitary Tensor-ESPRIT can be applied to derive estimates of the AoAs, AoDs and TdoAs.

\section{A. Data Model Reduction}

As stated previously, each antenna is described in terms of a polarimetric impulse response. To apply the Unitary Tensor-ESPRIT, the antenna's cross-polarimetric pattern is assumed as negligible. A single entry in $\mathbf{b}_{0}\left(\varphi^{R x}, \varphi^{T x}, \tau\right)$ remains, the others are zero. Hence, the data model in equation (9) reduces to

$$
\begin{aligned}
y^{\alpha, \beta} & \left(\varphi^{R x}, \varphi^{T x}, \tau\right) \\
& =m^{\alpha, \beta}\left(\varphi^{R x}, \varphi^{T x}, \tau\right)+n(\tau) \\
& =b_{0}^{\alpha, \beta}\left(\varphi^{R x}, \varphi^{T x}, \tau\right) * h^{\alpha, \beta}\left(\varphi^{R x}, \varphi^{T x}, \tau\right)+n(\tau)
\end{aligned}
$$

with the reduced propagation channel model

$$
\begin{aligned}
h^{\alpha, \beta} & \left(\varphi^{R x}, \varphi^{T x}, \tau\right) \\
& =\sum_{p=1}^{P} \gamma_{p}^{\alpha, \beta} \delta\left(\varphi^{R x}-\varphi_{p}^{R x}\right) \delta\left(\varphi^{T x}-\varphi_{p}^{T x}\right) \delta\left(\tau-\tau_{p}\right)
\end{aligned}
$$

and the reduced measurement system response

$$
b_{0}^{\alpha, \beta}\left(\varphi^{R x}, \varphi^{T x}, \tau\right)=b_{0, T x}^{\alpha}\left(\varphi^{R x}, \tau\right) * b_{0, R x}^{\beta}\left(\varphi^{T x}, \tau\right)
$$

Variables $\alpha$ and $\beta$ denote either $\varphi$ or $\vartheta$ co-polarisation.

Ignoring polarimetric antenna pattern introduces a mismatch between the measured data and the assumed data model. An incomplete data model impacts the solution of the inverse problem, which degrades the estimation quality [23], [24]. However, examination of this estimation degradation is conducted in Section V.

The tensor $\mathcal{Y}$ of polarimetric measurements is reshaped into the tensor $\mathcal{Y}_{\text {polar }} \in \mathbb{C}^{M_{r} \times M_{t} \times M_{f} \times 4}$, with the last dimension yielding the measurements of all polarisation combinations. 


\section{B. Beam-space Transformation and Calibration}

First, measurements with single polarised antennas are considered, denoted by $\mathcal{Y}_{\text {polar }}^{\alpha, \beta} \in \mathbb{C}^{M_{r} \times M_{t} \times M_{f}}$. Due to the rotation of a single antenna in the azimuth domain, the convolution in equation (3) is a cyclic convolution, because the azimuth is periodic. In case of a discretisation of the convolution result, the convolution can be described by a circulant matrix, constructed from a vector $\mathbf{b}$ by $\mathcal{C}(\mathbf{b})$. Considering a frequency slice $\mathbf{Y}(f) \in \mathbb{C}^{M_{r} \times M_{t}}$ of the tensor $\mathcal{Y}_{\text {polar }}^{\alpha, \beta}$ Fourier transformed in delay domain, the convolution in azimuth domain according to equation (24) is

$$
\mathbf{Y}(f)=\mathcal{C}\left(\mathbf{b}_{R x}^{\alpha}(f)\right) \cdot \mathbf{H}^{\alpha, \beta}(f) \cdot \mathcal{C}\left(\mathbf{b}_{T x}^{\beta}(f)\right)^{T}+\mathbf{n}(f)
$$

with $\mathbf{H}^{\alpha, \beta}(f)$ a frequency slice of the Fourier transformed and sampled propagation model according to equation (25). Vector $\mathbf{b}_{R x}^{\alpha}(f) \in \mathbb{C}^{M_{r} \times 1}$ and $\mathbf{b}_{T x}^{\beta}(f) \in \mathbb{C}^{M_{t} \times 1}$ contain the sampled antenna pattern at frequency $f$ and the respective antenna's rotation positions.

$$
\begin{aligned}
& \mathbf{b}_{R x}^{\alpha}(f)=\left[b_{0, R x}^{\alpha}(0, f) \ldots b_{0, R x}^{\alpha}\left(\left(M_{r}-1\right) \varphi_{0}^{R x}, f\right)\right]^{T} \\
& \mathbf{b}_{T x}^{\beta}(f)=\left[b_{0, T x}^{\beta}(0, f) \ldots b_{0, T x}^{\beta}\left(\left(M_{t}-1\right) \varphi_{0}^{T x}, f\right)\right]^{T}
\end{aligned}
$$

Facing the properties of circulant matrices, they are diagonalised by the Discrete Fourier Transform (DFT) matrix F [25].

$$
\mathcal{C}(\mathbf{b})=\mathbf{F}^{H} \cdot \operatorname{diag}\{\mathbf{F} \cdot \mathbf{b}\} \cdot \mathbf{F}
$$

Applying DFT to equation (27) yields

$$
\begin{aligned}
& \mathbf{F}_{M_{r}} \cdot \mathbf{Y}(f) \cdot \mathbf{F}_{M_{t}}^{T} \\
& \quad=\mathbf{G}_{R x}^{\alpha}(f) \mathbf{H}_{b s}^{\alpha, \beta}(f) \mathbf{G}_{T x}^{\beta}(f)^{T}+\mathbf{F}_{M_{r}} \mathbf{N}(f) \mathbf{F}_{M_{t}}^{T}
\end{aligned}
$$

with

$$
\begin{aligned}
\mathbf{H}_{b s}^{\alpha, \beta}(f) & =\mathbf{F}_{M_{r}} \cdot \mathbf{H}^{\alpha, \beta}(f) \cdot \mathbf{F}_{M_{t}}^{T} \\
\mathbf{G}_{R x}^{\alpha}(f) & =\operatorname{diag}\left\{\mathbf{F}_{M_{r}} \cdot \mathbf{b}_{R x}^{\alpha}(f)\right\} \\
\mathbf{G}_{T x}^{\beta}(f) & =\operatorname{diag}\left\{\mathbf{F}_{M_{t}} \cdot \mathbf{b}_{T x}^{\beta}(f)\right\}
\end{aligned}
$$

Matrix $\mathbf{H}_{b s}^{\alpha, \beta}(f)$ contains a frequency slice of the Fourier transformed and sampled propagation model in beam-space. In beam-space, a path arriving/departing from angle $\varphi_{p}$ results in a complex exponential $\exp \left(-\jmath 2 \pi l \mu_{0} \varphi_{p}\right)$, with $\mu_{0}=1 / 2 \pi$. The same holds for the beam-space (Fourier) transformed delay dimension as well, where the complex exponential is $\exp \left(-\jmath 2 \pi l f_{0} \tau_{p}\right)$, with $f_{0}=1 / M_{f} \tau_{0}$.

In order to describe the beam-space transformation for the complete measurement tensor $\mathcal{Y}_{\text {polar }}^{\alpha, \beta}$, the tensor's 1-mode unfolding with subsequent vectorisation is considered.

$$
\mathbf{y}^{\alpha, \beta}=\operatorname{vec}\left\{\left[\mathcal{Y}_{\text {polar }}^{\alpha, \beta}\right]_{(1)}\right\}
$$

The measurement's beam-space transformation is given by

$$
\mathbf{F} \cdot \mathbf{y}^{\alpha, \beta}=\mathbf{G}^{\alpha, \beta} \cdot \mathbf{h}_{b s}^{\alpha, \beta}+\mathbf{n}_{b s} .
$$

Vector $\mathbf{h}_{b s}^{\alpha, \beta}$ and $\mathbf{n}_{b s}$ denotes the sampled propagation model (25) and noise in beam-space, respectively. Matrix $\mathbf{F} \in \mathbb{C}^{L \times M}$ denotes the DFT matrix in the angle-delay domain

$$
\mathbf{F}=\mathbf{J}^{f} \mathbf{F}_{M_{f}} \otimes \mathbf{J}^{T x} \mathbf{F}_{M_{t}} \otimes \mathbf{J}^{R x} \mathbf{F}_{M_{r}}
$$

with $\mathbf{F}_{M_{f}} \in \mathbb{C}^{M_{f} \times M_{f}}, \mathbf{F}_{M_{r}} \in \mathbb{C}^{M_{r} \times M_{r}}, \mathbf{F}_{M_{t}} \in \mathbb{C}^{M_{t} \times M_{t}}$ being the Fourier-transformation matrices in delay and azimuth domains. Matrices $\mathbf{J}^{f} \in \mathbb{R}^{L_{f} \times M_{f}}, \mathbf{J}^{R x} \in \mathbb{R}^{L_{r} \times M_{r}}$, $\mathbf{J}^{T x} \in \mathbb{R}^{L_{t} \times M_{t}}$ are selection matrices, explained later on. Matrix $\mathbf{G}^{\alpha, \beta} \in \mathbb{C}^{L \times L}$ is the sampled beam-space response of the measurement system.

$$
\mathbf{G}^{\alpha, \beta}=\operatorname{diag}\left\{\operatorname{vec}\left\{\left(\mathbf{J}^{T x} \mathbf{G}_{T x}^{\beta} \mathbf{J}^{f^{T}}\right) \diamond\left(\mathbf{J}^{R x} \mathbf{G}_{R x}^{\alpha} \mathbf{J}^{f^{T}}\right)\right\}\right\}
$$

with matrix $\mathbf{G}_{R x}^{\alpha} \in \mathbb{C}^{M_{r} \times M_{f}}$ and $\mathbf{G}_{T x}^{\beta} \in \mathbb{C}^{M_{t} \times M_{f}}$ being the sampled and beam-space transformed antenna pattern at $\mathrm{Rx}$ and $\mathrm{Tx}$ side for the respective polarisation.

$$
\begin{aligned}
& \mathbf{G}_{R x}^{\alpha}=\mathbf{F}_{M_{r}} \cdot\left[\mathbf{b}_{R x}^{\alpha}(0) \ldots \mathbf{b}_{R x}^{\alpha}\left(\left(M_{f}-1\right) f_{0}\right)\right] \\
& \mathbf{G}_{T x}^{\beta}=\mathbf{F}_{M_{t}} \cdot\left[\mathbf{b}_{T x}^{\beta}(0) \ldots \mathbf{b}_{T x}^{\beta}\left(\left(M_{f}-1\right) f_{0}\right)\right]
\end{aligned}
$$

In order to get an estimate $\hat{\mathbf{h}}_{b s}^{\alpha, \beta}$ of the sampled propagation model in beam-space, calibration of the beam-space transformed measurements is conducted.

$$
\hat{\mathbf{h}}_{b s}^{\alpha, \beta}=\mathbf{G}^{\alpha, \beta^{-1}} \cdot \mathbf{F} \cdot \mathbf{y}=\mathbf{h}_{b s}^{\alpha, \beta}+\mathbf{G}^{\alpha, \beta^{-1}} \cdot \mathbf{n}_{b s}
$$

Considering the tensor of polarimetric measurements $\mathcal{Y}_{\text {polar }}$, define his 4-mode unfolding

$$
\mathbf{Y}=\left[\mathcal{Y}_{\text {polar }}\right]_{(4)}^{T}=\left[\mathbf{y}^{\varphi, \varphi} \mathbf{y}^{\varphi, \vartheta} \mathbf{y}^{\vartheta, \varphi} \mathbf{y}^{\vartheta, \vartheta}\right]
$$

and utilising equation (36) for transformation and calibration yields beam-space estimates for all 4 polarisation combinations.

$$
\begin{aligned}
& \hat{\mathbf{h}}_{b s}^{\varphi, \varphi}=\mathbf{G}^{\varphi, \varphi^{-1}} \cdot \mathbf{F} \cdot \mathbf{y}^{\varphi, \varphi} \\
& \hat{\mathbf{h}}_{b s}^{\varphi, \vartheta}=\mathbf{G}^{\varphi, \vartheta^{-1}} \cdot \mathbf{F} \cdot \mathbf{y}^{\varphi, \vartheta} \\
& \hat{\mathbf{h}}_{b s}^{\vartheta, \vartheta}=\mathbf{G}^{\vartheta, \vartheta^{-1}} \cdot \mathbf{F} \cdot \mathbf{y}^{\vartheta, \vartheta} \\
& \hat{\mathbf{h}}_{b s}^{\vartheta, \varphi}=\mathbf{G}^{\vartheta, \varphi^{-1}} \cdot \mathbf{F} \cdot \mathbf{y}^{\vartheta, \varphi}
\end{aligned}
$$

These vectors are concatenated in matrix $\mathbf{H}_{b s}$.

$$
\mathbf{H}_{b s}=\left[\begin{array}{lll}
\hat{\mathbf{h}}_{b s}^{\varphi, \varphi} & \hat{\mathbf{h}}_{b s}^{\varphi, \vartheta} & \hat{\mathbf{h}}_{b s}^{\vartheta, \varphi} \hat{\mathbf{h}}_{b s}^{\vartheta, \vartheta}
\end{array}\right]
$$

\section{Beam-space Data Model}

The data model in the calibrated beam-space is

$$
\mathbf{H}_{b s}=\mathbf{A}\left(\boldsymbol{\tau}, \varphi^{T x}, \varphi^{R x}\right) \cdot \boldsymbol{\gamma}+\mathbf{N}_{b s} .
$$

Matrix $\boldsymbol{\Gamma}=\left[\gamma^{\varphi, \varphi} \gamma^{\varphi, \vartheta} \gamma^{\vartheta, \varphi} \gamma^{\vartheta, \vartheta}\right]$ contains the polarimetric path weights and matrix $\mathbf{A}\left(\boldsymbol{\tau}, \varphi^{T x}, \varphi^{R x}\right) \in \mathbb{C}^{M \times P}$ contains the exponentials according to the angles and delays for each path.

$$
\begin{aligned}
& \mathbf{A}\left(\boldsymbol{\tau}, \varphi^{T x}, \varphi^{R x}\right)=\mathbf{A}(\boldsymbol{\tau}) \diamond \mathbf{A}\left(\varphi^{T x}\right) \diamond \mathbf{A}\left(\varphi^{R x}\right) \\
& \mathbf{A}(\boldsymbol{\tau})=\exp \left(-\jmath 2 \pi f_{0} \cdot \mathbf{l}_{f} \cdot \boldsymbol{\tau}^{T}\right) \quad \in \mathbb{C}^{L_{f} \times P} \\
& \mathbf{A}\left(\boldsymbol{\varphi}^{R x}\right)=\exp \left(-\jmath 2 \pi \mu_{0} \cdot \mathbf{l}_{r} \cdot \varphi^{R x^{T}}\right) \in \mathbb{C}^{L_{r} \times P} \\
& \mathbf{A}\left(\varphi^{T x}\right)=\exp \left(-\jmath 2 \pi \mu_{0} \cdot \mathbf{l}_{t} \cdot \varphi^{T x^{T}}\right) \in \mathbb{C}^{L_{t} \times P} \\
& \mathbf{l}_{r}=\left[0 \ldots L_{r}-1\right]^{T} \\
& \mathbf{l}_{f}=\left[0 \ldots L_{f}-1\right]^{T} \\
& \mathbf{l}_{t}=\left[0 \ldots L_{t}-1\right]^{T}
\end{aligned}
$$

Estimation of the path's AoA, AoD and TdoA based on the data model in equation (40) is a 3-dimensional harmonic retrieval problem. 


\section{Harmonic Retrieval and Path Weight Estimation}

Reshaping matrix $\mathbf{H}_{b s}$ yields the tensor $\boldsymbol{H}_{b s} \in \mathbb{C}^{L_{r} \times L_{t} \times L_{f} \times 4}$, which is given by

$$
\boldsymbol{H}_{b s}=\boldsymbol{I} \times{ }_{1} \mathbf{A}\left(\varphi^{R x}\right) \times{ }_{2} \mathbf{A}\left(\varphi^{T x}\right) \times{ }_{3} \mathbf{A}(\boldsymbol{\tau}) \times{ }_{4} \boldsymbol{\Gamma}^{T}
$$

Tensor $\boldsymbol{I} \in \mathbb{C}^{P \times P \times P \times P}$ denotes the identity tensor. The first three dimensions of tensor $\boldsymbol{H}_{b s}$ feature shift invariance and centro-Hermitian property. Thus the Unitary Tensor-ESPRIT becomes applicable for estimation of $\tau, \varphi^{R x}$ and $\varphi^{T x}$ [15], [16].

After inferring estimates for AoA, AoD and TdoA, the matrix of polarimetric path weights can be estimated by the best-linear-unbiased estimator (BLUE) [21]

$$
\hat{\boldsymbol{\gamma}}=\left(\hat{\mathbf{A}}^{H} \boldsymbol{\Sigma}^{-1} \hat{\mathbf{A}}\right)^{-1} \hat{\mathbf{A}}^{H} \boldsymbol{\Sigma}^{-1} \cdot \mathbf{H}_{b s}
$$

whereas $\hat{\mathbf{A}}=\mathbf{A}\left(\hat{\varphi}^{T x}, \hat{\varphi}^{R x}, \hat{\boldsymbol{\tau}}\right)$. Due to the transformation according to equation (36), the noise's second-order statistic is varied, which is differently for each polarisation combination $\alpha, \beta$. Hence, the BLUE has to be adapted accordingly

$$
\hat{\boldsymbol{\gamma}}^{\alpha, \beta}=\left(\hat{\mathbf{A}}^{H} \boldsymbol{\Sigma}^{\alpha, \beta^{-1}} \hat{\mathbf{A}}\right)^{-1} \hat{\mathbf{A}}^{H} \boldsymbol{\Sigma}^{\alpha, \beta^{-1}} \cdot \hat{\mathbf{h}}_{b s}^{\alpha, \beta}
$$

with $\boldsymbol{\Sigma}^{\alpha, \beta}=\mathbf{G}^{\alpha, \beta^{-1}} \mathbf{F} \boldsymbol{\Sigma} \mathbf{F}^{H} \mathbf{G}^{\alpha, \beta^{-H}}$ the modified noise covariance matrix for each polarisation combination.

\section{E. Sampling Step Size and Selection Matrices}

The multi-dimensional convolution in equation (24) is sampled by the measurement system. A process is observed in each dimension, featuring a bandwidth which is related to the system bandwidth in the respective domain. Hence, in order to avoid aliasing, the Nyquist criterion has to be fulfilled by proper selection of the sampling step sizes $\varphi_{0}^{T x}, \varphi_{0}^{R x}$ and $\tau_{0}$. A larger bandwidth increase the (Rayleigh) resolution, to the cost of necessary denser sampling.

The process' bandwidth related to the delay domain is determined by the sounding signal bandwidth. Hence, the delay domain sampling step size $\tau_{0}$ has to be chosen accordingly at the receiver.

The process' bandwidth in the spatial domain is related to the array aperture, which depends on the array radius and the antenna pattern. The bandwidth will be increased, if high gain antennas are utilised and/or the antenna is rotated on a large circumference. Hence, smaller step sizes $\varphi_{0}^{T x}$ and $\varphi_{0}^{R x}$ have to be chosen. Selection of the sampling step size under practical considerations can be tough due to e.g. mechanical limitations, such that a certain array radius is hard to guarantee. Hence, calibration measurements of the virtual UCA are necessary, in order to investigate the spatial bandwidth and to deduce the required sampling step size [26].

The step sizes has to be chosen, such that a band limited process is present in the dimension's beam-space. Considering equation (36) and a band limited process, SNR problems occur if calibration is carried out. Hence, it is necessary to select signal areas in the beam-space featuring enough SNR for proper calibration. This selection is described by the matrices $\mathbf{J}^{f} \in \mathbb{R}^{L_{f} \times M_{f}}, \mathbf{J}^{R x} \in \mathbb{R}^{L_{r} \times M_{r}}, \mathbf{J}^{T x} \in \mathbb{R}^{L_{t} \times M_{t}}$, which

\begin{tabular}{|c|c|c|c|}
\hline AoA & $-5^{\circ}$ & $0^{\circ}$ & $5^{\circ}$ \\
\hline AoD & $5^{\circ}$ & $0^{\circ}$ & $-5^{\circ}$ \\
\hline TdoA & $25 \mathrm{~ns}$ & $25.5 \mathrm{~ns}$ & $26.5 \mathrm{~ns}$ \\
\hline Bandwidth & \multicolumn{3}{|c|}{$1 \mathrm{GHz}$} \\
\hline Center frequency & \multicolumn{3}{|c|}{$71.5 \mathrm{GHz}$} \\
\hline UCA radius & \multicolumn{3}{|c|}{$20 \mathrm{~mm}$} \\
\hline Antennas & \multicolumn{3}{|c|}{ Dual-polarimetric horn antenna [26] } \\
\hline$M_{f}$ & \multicolumn{3}{|c|}{101} \\
\hline$M_{r}^{\prime}$ & \multicolumn{3}{|c|}{45} \\
\hline$M_{t}$ & \multicolumn{3}{|c|}{45} \\
\hline No. Monte-Carlo runs & \multicolumn{3}{|c|}{1000} \\
\hline
\end{tabular}
entries are 1 to select a signal area and 0 elsewhere.
TABLE I: Simulation settings

\section{Simulations}

In order to compare the ML and beam-space ESPRIT estimator, their root-mean-square error (RMSE) for various noise powers is calculated from Monte-Carlo simulations. The simulation settings are summarised in Table I. The number of spatial sampling points $M_{r}$ and $M_{t}$ to fulfil the Nyquist criterion was deduced empirically.

Synthetic measurement data are generated from equation (14), based on known parameter values. The parameters AoA, AoD and TdoA can be chosen randomly, but the polarimetric path weights not necessarily. Generally, the path weight's magnitude correspondence to the transmission attenuation. For a single bounce reflection, the bi-static radar equation can be utilised to calculate this attenuation according to a given bi-static range $c \tau=R_{R x}+R_{T x}$, the polarimetric radar cross section (RCS) $\rho^{\alpha, \beta}$ and the centre frequency's wavelength $\lambda_{c}$ [27]

$$
\gamma^{\alpha, \beta}=\sqrt{\frac{\lambda_{c}^{2} \cdot \rho^{\alpha, \beta}}{(4 \pi)^{3} \cdot R_{R x}^{2} \cdot R_{T x}^{2}}} e^{-\jmath 2 \pi\left(R_{T x}+R_{R x}\right) \lambda_{c}^{-1}} .
$$

For the conducted simulations, the polarimetric RCS is set to $\rho^{\alpha=\beta}=-30 \mathrm{~dB}$ and $\rho^{\alpha \neq \beta}=-45 \mathrm{~dB}$, and the bi-static range is $R_{R x}=R_{T x}=c \tau / 2$. Furthermore, the model order (number of paths $P$ ) is assumed as known.

The calculated RMSEs for the ML and beam-space ESPRIT are depicted in Fig. 2. Note, that the estimated parameters are normalised to $2 \pi$. Additionally, the Cramér-Rao lower bound (CRLB) is shown, indicating the lowest achievable RMSE for any unbiased estimator [21]. As becomes visible, the RMSE of the beam-space ESPRIT does not achieve the CRLB, indicating that the estimator is not efficient. On the other hand, the ML estimator achieves the CRLB and thus is considered as an efficient estimator. Because the beamspace ESPRIT assumes a simplified data model and the bandwidth was reduced due to the beam-space transformation, the estimation performance is naturally degraded compared to the ML estimator. Furthermore, for noise powers in the range from $30 \mathrm{~dB}$ to $40 \mathrm{~dB}$, the RMSE curves of the beamspace ESPRIT do not show a settling behaviour. Therefore, additional simulations have been carried out considering a single path only, with the following parameter settings: AoA $0^{\circ}$, AoD $0^{\circ}$, TdoA $25.5 \mathrm{~ns}$. The calculated RMSEs are depicted in Fig. 3. Obviously, the curves of the beam-space ESPRIT do not show the settling. Hence, the settling is assumed to result from the 3 path scenario setting. 

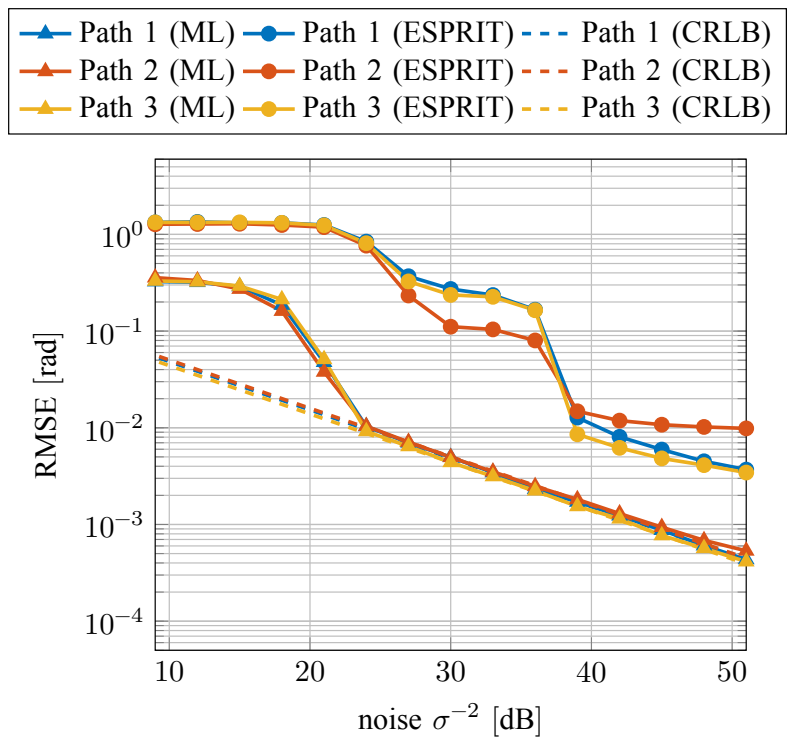

(a) Azimuth of arrival (AoA)

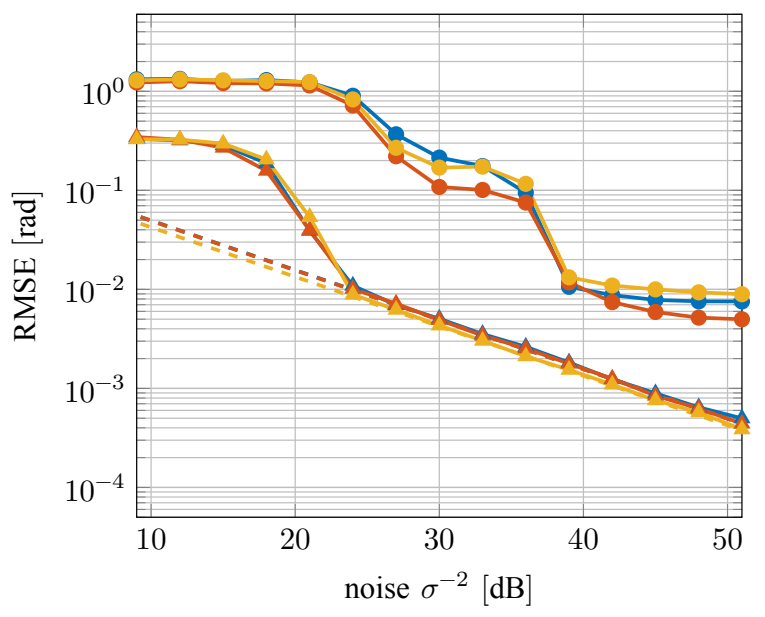

(b) Azimuth of departure (AoD)

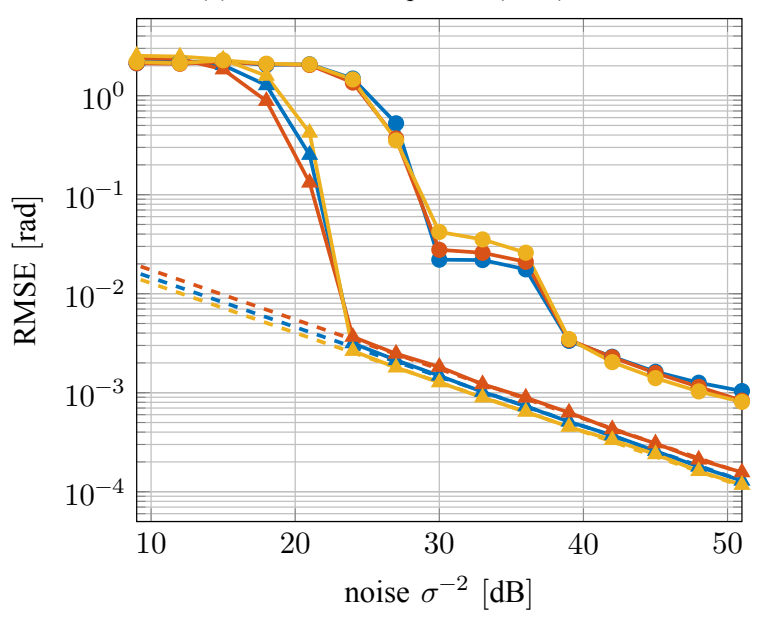

(c) Time difference of arrival (TdoA)

Fig. 2: RMSE of parameter estimates in comparison to the CRLB for triple path scenario. Parameter estimates are normalised to $2 \pi$

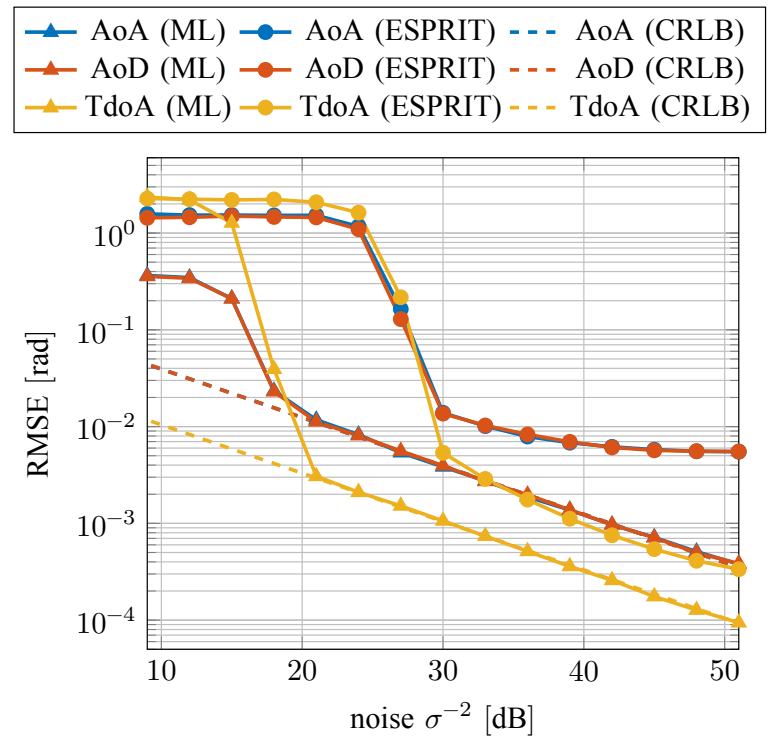

Fig. 3: RMSE of parameter estimates in comparison to the CRLB for single path scenario. Parameter estimates are normalised to $2 \pi$

\section{Conclusion}

In this paper, system identification in conjunction with high resolution parameter estimation was motivated. The physical system under identification is the propagation channel, which is sampled by a MIMO system in spatial and delay domain. Here, sampling of the spatial domain (angle domain) by rotating antennas at $\mathrm{Tx}$ and $\mathrm{Rx}$ side has been considered. Parameters of a propagation model are subsequently estimated from the sampling data, with a resolution higher than the Rayleigh resolution limit. A data model based on the multi-dimensional convolution of the propagation model and the measurement system model was derived, describing the incorporation of the propagation parameters in the sampling data. The data model is required to solve the inverse problem, which arises due to the parameter estimation task. A solver for the inverse problem has been proposed, which was derived from the maximum-likelihood principle.

Applying beam-space transformation and subsequent calibration, the parameter estimation task transforms into a multidimensional harmonic retrieval problem. This problem can be solved by the Unitary Tensor-ESPRIT, which is computationally simpler than the enumerative-search based ML estimator. Beam-space pre-processing requires a band limited process in all dimensions. Hence, the sampling step size in angle and delay domain has to be chosen in accordance to the Nyquist criterion. Also, the complete beam-space is commonly not usable for parameter estimation, because SNR problems occur due to the presence of band limited processes. Besides its computational simplicity in comparison to the ML estimator, the beam-space ESPRIT assumes simplifications of the data model. The full-polarimetric antenna pattern is not incorporated in the estimator. Only the co-polar pattern is considered and the cross-polar pattern is neglected. Hence, a large cross-polar discrimination is assumed, which is difficult to guarantee in real applications. However, this simplification introduces a mismatch between measurements and the data 
model, which degrades the quality of the parameter estimates.

Monte-Carlo simulations using synthetic data were carried out, in order to compare the ML and beam-space ESPRIT in terms of the achievable RMSE for increasing SNR. It turned out, that the beam-space ESPRIT is not an efficient estimator, because the Cramér-Rao lower bound is not attained for increasing SNR. On the other hand, the ML based estimator attends the CRLB and therefore outperforms the beam-space ESPRIT in terms of the achievable RMSE.

\section{Acknowledgment}

Stephan Häfner is funded by the Deutsche Forschungsgemeinschaft (DFG) under grant no. 317632307.

\section{References}

[1] R. Thomä, M. Landmann, A. Richter, and U. Trautwein, "Multidimensional High-Resolution Channel Sounding Measurement," in Smart Antennas - State of the Art, ser. EURASIP Book Series on Signal Processing and Communications. Hindawi, 2005, vol. 3.

[2] M. Steinbauer, A. Molisch, and E. Bonek, "The Double-Directional Radio Channel," IEEE Antennas and Propagation Magazine, vol. 43, no. 4, pp. 51-63, Aug 2001.

[3] S. Häfner, D. A. Dupleich, R. Müller et al., "Characterisation of Channel Measurements at $70 \mathrm{GHz}$ in Indoor Femtocells," in 81st IEEE Vehicular Technology Conference (VTC Spring), May 2015, pp. 1-5.

[4] D. Dupleich, S. Häfner, C. Schneider et al., "Double-directional and dual-polarimetric indoor measurements at $70 \mathrm{GHz}$," in 26th IEEE Annual International Symposium on Personal, Indoor, and Mobile Radio Communications (PIMRC), Aug 2015, pp. 2234-2238.

[5] A. Richter and R. S. Thomä, "CUBA-ESPRIT for Angle Estimation with Circular Uniform Beam Arrays," in Millennium Conference on Antennas \& Propagation, April 2000.

[6] M. Haardt, R. Thomä, and A. Richter, "Multidimensional HighResolution Parameter Estimation with Applications to Channel Sounding," in High-Resolution and Robust Signal Processing. Marcel Dekker, Inc., 2004.

[7] J. Liang, M. D. Kim, J. Lee et al., "Comparison of SAGE and BBF for Multipath Angular and Delay Spread Characteristics with $28 \mathrm{GHz}$ Channel Measurements," in IEEE-APS Topical Conference on Antennas and Propagation in Wireless Communications (APWC), Sept 2016.

[8] H. A. Nguyen, K. Mahler, M. Peter et al., "Estimation of DoA Based on Large-scale Virtual Array Data," in 10th European Conference on Antennas and Propagation (EuCAP), April 2016, pp. 1-4.

[9] Y. Ji, X. Yin, H. Wang et al., “Antenna-De-embedded Characterization for 13-17 GHz Wave Propagation in Indoor Environments," IEEE Antennas and Wireless Propagation Letters, vol. PP, no. 99, 2016.

[10] X. Lu, R. Zhang, Y. Zhou et al., "Convolutional Modeling and Antenna De-Embedding for Wideband Spatial mmWave Channel Measurement," in IEEE Wireless Communications and Networking Conference (WCNC), March 2017.

[11] F. Zhang, W. Fan, and G. F. Pedersen, "Frequency-Invariant Uniform Circular Array for Wideband mm-Wave Channel Characterization,' IEEE Antennas and Wireless Propagation Letters, vol. 16, 2017.

[12] R. Boyer, "Decoupled root-MUSIC algorithm for Multidimensional Harmonic retrieval," in 9th IEEE Workshop on Signal Processing Advances in Wireless Communications, July 2008, pp. 16-20.

[13] M. Pesavento, C. F. Mecklenbräuker, and J. F. Böhme, "Multidimensional Rank Reduction Estimator for Parametric MIMO Channel Models," EURASIP Journal on Advances in Signal Processing, vol. 2004, no. 9, p. 839148, Aug 2004.

[14] J. P. C. L. da Costa, F. Roemer, M. Weis, and M. Haardt, "Robust R-D Parameter Estimation via Closed-Form PARAFAC," in 14th International ITG Workshop on Smart Antennas (WSA), Feb 2010, pp. 99-106.

[15] M. Haardt, F. Roemer, and G. D. Galdo, "Higher-Order SVD-Based Subspace Estimation to Improve the Parameter Estimation Accuracy in Multidimensional Harmonic Retrieval Problems," IEEE Transactions on Signal Processing, vol. 56, no. 7, pp. 3198-3213, July 2008.

[16] F. Roemer, M. Haardt, and G. D. Galdo, "Analytical Performance Assessment of Multi-Dimensional Matrix- and Tensor-Based ESPRITType Algorithms," IEEE Transactions on Signal Processing, vol. 62, no. 10, pp. 2611-2625, May 2014.
[17] R. Thomä, M. Landmann, G. Sommerkorn et al., "Multidimensional High-Resolution Channel Sounding in Mobile Radio," in 21st IEEE Instrumentation and Measurement Technology Conference (IMTC), vol. 1, May 2004, pp. 257-262 Vol.1.

[18] P. Eggers, "Super resolution and deconvolution of angular power spectra," in 8th IEEE International Symposium on Personal, Indoor and Mobile Radio Communications (PIMRC), vol. 3, Sep 1997, pp. 801-805.

[19] P. Schreier and L. Scharf, Statistical Signal Processing of ComplexValued Data: The Theory of Improper and Noncircular Signals. Cambridge University Press, 2010.

[20] L. D. Lathauwer, B. D. Moor, and J. Vandewalle, “A multilinear Singular Value Decomposition," SIAM J. Matrix Anal. Appl, vol. 21, 2000.

[21] H. L. Van Trees, Optimum Array Processing - Detection, Estimation, and Modulation Theory. New York: John Wiley \& Sons, 2004.

[22] S. Häfner, S. Röding, G. Sommerkorn et al., "Contribution to Drone Detection by Exploiting Parameter Estimation for a Prototype mm-Wave Radar System," in 22th International ITG Workshop on Smart Antennas, March 2018.

[23] M. Landmann, M. Käske, and R. Thomä, "Impact of Incomplete and Inaccurate Data Models on High Resolution Parameter Estimation in Multidimensional Channel Sounding," IEEE Transactions on Antennas and Propagation, vol. 60, no. 2, pp. 557-573, Feb 2012.

[24] S. Häfner, M. Käske, R. S. Thomä et al., "Selection of Antenna Array Configuration for Polarimetric Direction Finding in Correlated Signal Environments," in 19th International ITG Workshop on Smart Antennas, Mar 2015, pp. 1-8.

[25] K. B. Petersen and M. S. Pedersen, The Matrix Cookbook, 20121115th ed. Technical University of Denmark, Nov 2012. [Online]. Available: http://www2.imm.dtu.dk/pubdb/p.php?3274

[26] S. Häfner, R. Müller, and R. S. Thomä, "Full 3D Antenna Pattern Interpolation Using Fourier Transform Based Wavefield Modelling," in 20th International ITG Workshop on Smart Antennas, March 2016.

[27] M. I. Skolnik, Radar Handbook, 2nd ed. New York: McGraw Hill, 1990. 